\title{
Metastatic cutaneous epithelioid hemangioendothelioma involving right atrium - a case report
}

\author{
Sandy Mpho Mosenye ${ }^{1}$, Rahul Daimari ${ }^{1,2}$, Neha Sharma ${ }^{1}$, Princess Lelani Chikanda ${ }^{3}$, Feng Zhou ${ }^{4}$, Godfrey \\ Mutashambara Rwegerera*1,2 \\ ${ }^{1}$ Department of Medicine, Princess Marina Hospital, Gaborone, Botswana \\ ${ }^{2}$ Department of Internal Medicine, University of Botswana, Gaborone, Botswana \\ ${ }^{3}$ Faculty of Medicine, University of Botswana, Gaborone, Botswana \\ ${ }^{4}$ Department of Pathology, National Health Laboratory, Gaborone, Botswana
}

Received: May 22, 2016

DOI: $10.5430 /$ crim.v3n3p47
Accepted: June 20, 2016

Online Published: June 23, 2016

URL: http://dx.doi.org/10.5430/crim.v3n3p47

\begin{abstract}
Epithelioid hemangioendothelioma (EHE) is a rare vascular endothelial tumour with unpredictable malignant potential. The common sites involved are the lung, liver and soft tissues; with cardiac involvement being a very rare occurrence. The diagnosis of this rare vascular tumor is made by typical histopathological findings and confirmed by specific immunohistochemistry. We present a case of a patient with a long standing painless scalp lesion who presented with pulmonary symptoms and ultimately diagnosed to have metastatic cutaneous EHE involving the lungs, pericardium and right atrium.
\end{abstract}

Key Words: Epithelioid hemangioendothelioma, Scalp, Pulmonary, Right atrium

\section{INTRODUCTION}

Epithelioid hemangioendothelioma (EHE) is a rare malignant vascular neoplasm of endothelial origin, with intermediate malignant potential ranging between benign hemangioma and angiosarcoma. ${ }^{[1-4]}$ The estimated prevalence of epithelioid hemangioendothelioma is less than 1 in 1 million. ${ }^{[5]}$ The natural history of EHE is extremely unpredictable, and there is substantial interpatient variability in disease progression with reported potential to metastasize even after a very long period. ${ }^{[6,7]}$ Clinical presentation of EHE usually involves a painful mass in superficial or deep soft tissue, with primary lesions also occurring in other organs such as lung, liver and bones. ${ }^{[8]}$ Cardiac involvement by primary
EHE is extremely rare. ${ }^{[7,9,10]}$ Diagnosis of EHE is made on histology, requiring immunohistochemical staining for markers specific to vascular endothelium. ${ }^{[1]}$ Characteristic histopathological features include;-vascular tumor characterized by solid nests and cords of atypical spindled endothelial cells, some of which have distinct intracellular lumina with moderate atypia and mitotic figures, and immunohistochemistry positive for CD34, CD31 and factor VIII confirms a diagnosis of EHE. ${ }^{[10]}$ We present this rare case of a young man who presented with painless scalp lesion for over 20 years, which latter progressed to pulmonary symptoms, with final diagnosis confirming metastasis of EHE to the lungs, pericardium and right atrium. We did not find cases in En-

\footnotetext{
* Correspondence: Godfrey Mutashambara Rwegerera; Email: grwege@yahoo.com; Address: Private Bag 00713, Faculty of Medicine, Univeristy of Botswana, Gaborone, Botswana.
} 
glish literature that involved metastasis to the right atrium apart from those reported to be of primary cardiac EHE.

\section{CASE REPORT}

A 31-year-old young man presented at Princess Marina Hospital, a referral hospital at the capital city Gaborone in Botswana in July 2015 with history of four months pleuritic central chest pain associated with progressive worsening shortness of breath. He reported history of loss of appetite and significant loss of weight, but denied history of low grade fevers, night sweats, cough or known TB contacts. He never smoked and denied ever drinking alcohol; he was electrician by profession with no prior history of working in the mines or industries where he might have been exposed to inhaled fumes or dusts. He had no family history of malignancy, diabetes mellitus, hypertension or any other known chronic diseases. He had been attended at local clinics and received numerous antibiotics for community acquired pneumonia without improvement, investigations prior to referral included sputum for acid fast bacilli which was negative and chest $\mathrm{x}$-ray revealed multiple bilateral nodular opacities (see Figure 1).

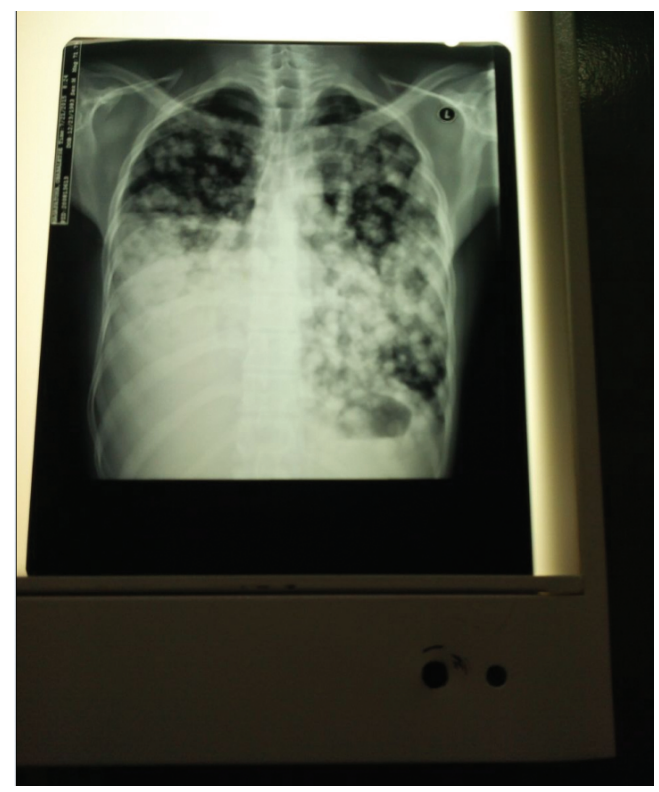

Figure 1. Chest $\mathrm{x}$-ray showing bilateral multiple nodular opacities

Bronchoscopy performed did not reveal any intraluminal lesions or any accessible extraluminal lesions. Initial examination on admission at Princess Marina Hospital revealed a chronically sick looking patient, in mild respiratory distress who was tachypnoeic at 24 breaths per minute, saturating at $94 \%$ on room air, blood pressure of $133 / 85 \mathrm{mmHg}$ and was tachycardic at 113 beats per minute. He was however afebrile
(Temperature of $36.5^{\circ} \mathrm{C}$ ), mildly pale, no finger clubbing, neither was he icteric nor cyanosed and he did not have any palpable lymph nodes. Respiratory examination revealed decreased chest movements and expansion on the right lung with dull percussion note on the right middle and lower zones, there was decreased air entry but no crepitations bilaterally. Cardiovascular system was essentially normal except for tachycardia. The rest of system examinations including neurological, musculoskeletal and abdominal were also noncontributory. On the background of a chest x-ray with multiple bilateral nodular lesions, a possibility of metastatic lung disease was considered; hence thorough examination of common sites for primary malignancy including thyroid and testes was performed and found to be normal. For skin examination, it was essentially normal except for a non-tender spherical swelling on frontal-central aspect of his head which was same dark colour as patient's rest of skin, the swelling had smooth edges and it measured $1.5 \mathrm{~cm}$ by $2 \mathrm{~cm}$ in diameter (further questioning of the scalp swelling, patients admitted to have noted the painless swelling since when he was young, over 20 years ago, the swelling was initially very small in size like a birth mark, he noted increase in size of the swelling over the past 9 months prior to admission).

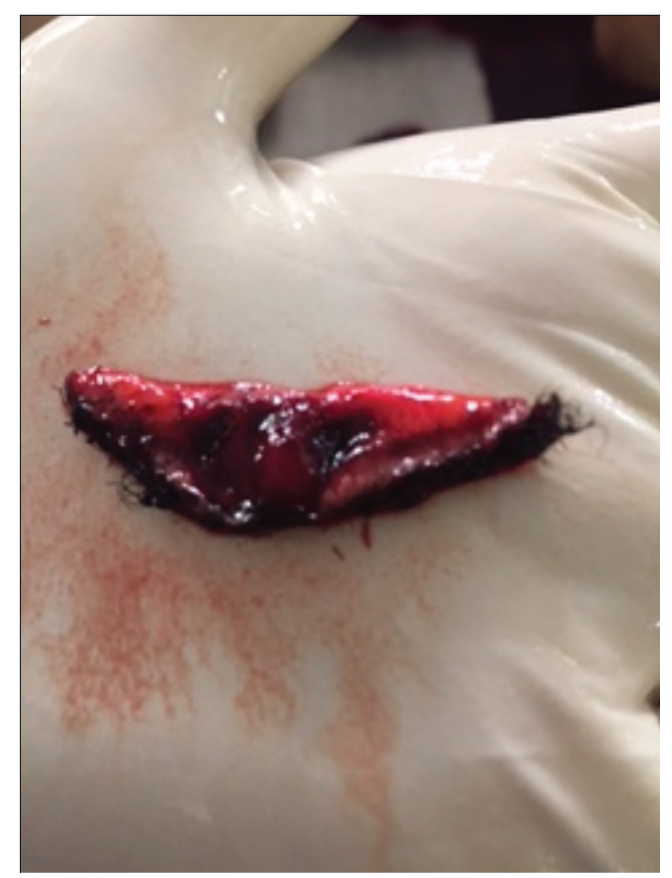

Figure 2. Gross anatomy of cut-section of brown scalp lesion

Work-up of the patient included baseline investigations; Full blood count was consistent with Iron deficiency anaemia (Haemoglobin of $9.3 \mathrm{~g} / \mathrm{dl}$, mean corpuscular volume of 79.4 $\mathrm{fl}$ ), the rest of white blood cell count (total and differential) and platelet count was unremarkable. Liver function 
tests/enzymes and renal function tests including electrolytes (calcium, phosphate, potassium, sodium and magnesium) were all within normal ranges.

Results of serum tumor markers were as follows: Carcinoembryonic antigen $(\mathrm{CEA})=1.07 \mathrm{ng} / \mathrm{ml}$ (Normal range $<2.0 \mathrm{ng} / \mathrm{ml}$ ), Serum Beta Human chorionic gonadotropin (HCG) $<1.2 \mathrm{mIU} / \mathrm{ml}$ (Normal range $<5 \mathrm{mIU} / \mathrm{ml}$ ), Alpha Fetoproteins $<0.5 \mathrm{IU} / \mathrm{ml}$ (Normal $=0-20 \mathrm{IU} / \mathrm{ml}$ ). Other blood tests included Human Immunodeficiency Virus (HIV) which was negative and elevated Lactate dehydrogenase $=872 \mathrm{U} / \mathrm{L}$ $($ Normal $=60-160 \mathrm{U} / \mathrm{L})$.

Computed tomography (CT-Chest and Abdomen) revealed multiple widespread hypodense ring-enhancing lesions of different sizes in lungs, significant irregular bilateral pleural thickening, and a homogenous hyperdensity in the left lower lobe of lung displacing the heart. Another large heterogeneous irregular mass predominantly hypodense necrosis/haemorrhage (density $27 \mathrm{Hu}$ ) suggesting left lower lobe mass extending medially and between the Inferior vena cava (IVC) and Aorta. Medially a more homogenous hyperdense mass suggested infiltration.

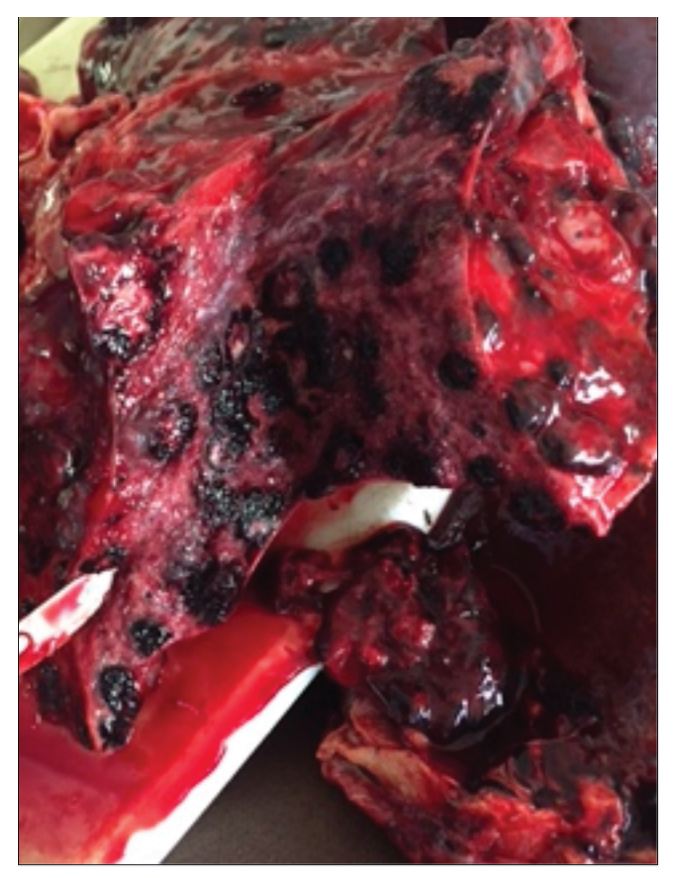

Figure 3. Gross anatomy of lung tissue showing diffuse multiple brown masses with haemorrhage and necrosis

There was partial encasement and contralateral displacement of the Heart and major vessels. For the abdomen, both liver and spleen was homogenous and not enlarged, gall bladder appeared normal and both renal outlines were of normal size, shape and positions with prompt perfusions, adrenal glands were not enlarged. The conclusion was of metastatic Published by Sciedu Press lung disease probably involving the heart. Ultrasonography scans for both thyroid and testicles was normal making the two primaries unlikely. Fine-needle aspiration cytology (FNAC) under ultrasound guidance was attempted and revealed mostly blood, inflammatory cells including lymphocytes and fibroblasts; the aspirate stained negative for acid fast bacilli, malignancy could not be ruled due to the scanty nature of specimen obtained. The patient condition worsened over the course of the next two weeks as he became more dyspnoeic and required oxygen therapy, with efforts in place to do further work-up; he became very hypoxic, had cardiac arrest and died despite resuscitation. Counseling was done to the family members who provided consent for the autopsy to be done.

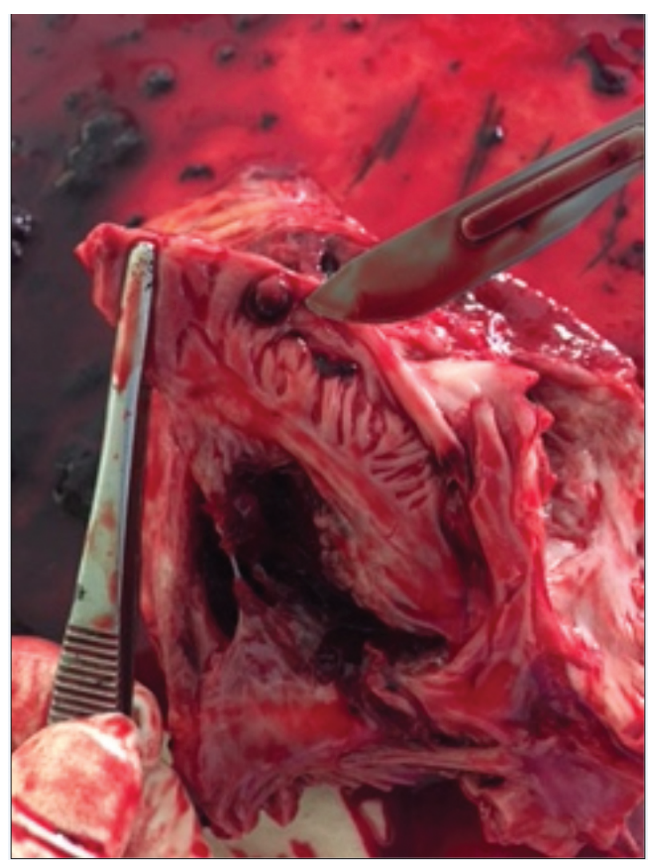

Figure 4. Gross anatomy showing tumor in the right atrium of the heart

The findings of the autopsy were as follows: Grossly, there was ulcerated nodule on the scalp measuring $2 \mathrm{~cm} \times 2 \mathrm{~cm}$ and cross section showed brown solid soft tissue (see Figure 2). Both lungs showed diffuse multiple brown masses with haemorrhage and necrosis (see Figure 3). The pericardium was involved by the lung mass and adhesive to the lung tissue with fibrosis and haemorrhage. There was a tumour measuring $8 \mathrm{~cm}$ in the largest diameter in the right atrium (see Figure 4). Histology of the autopsy specimen revealed a scalp tissue with a vascular tumour composed of vascular channels with atypical endothelium, compatible with epithelioid hemangioendothelioma (see Figure 5). Both lungs showed diffuse infiltration by a vasoproliferative and lung masses were composed of various sizes of vascular channels with haemor- 
rhage, thrombosis and necrosis. Some areas of the tumour cells were arranged in sheets (see Figure 6). The histological features favored an epithelioid hemangioendothelioma. The pericardium and the right atrium histology of the lesions were also consistent with metastatic lesions of epithelioid hemangioendothelioma in origin. Involvement of other vital organs such as brain, liver, spleen and kidneys was ruled out at histology. Immunohistochemistry of the biopsied specimens was positive for both CD34 and CD31. The conclusion was of a malignant disseminated cutaneous epithelioid hemangioendothelioma involving lungs, pericardium and right atrium.

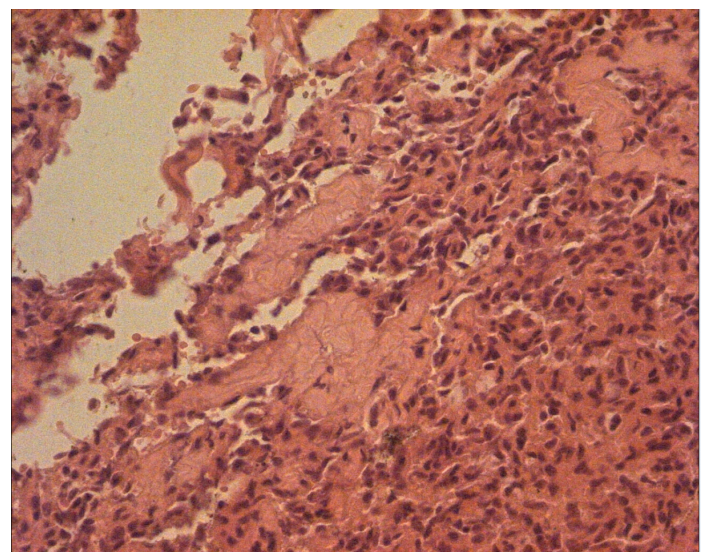

Figure 5. Scalp histology showing vascular spaces with area of solid nests of rounded to slightly spindled endothelial cells

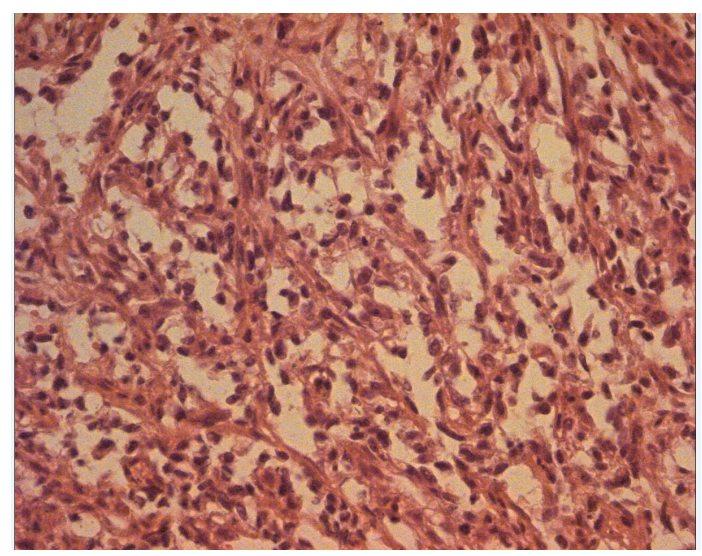

Figure 6. Lung histology showing areas of vascular channels lined by atypical cuboidal endothelium

\section{Discussion}

Epithelioid hemangioendothelioma represents the most aggressive member of the hemangioendothelioma family of tumors. Previous reports classified EHE as an intermediate between hemangioma and angiosarcoma in terms of recurrence and metastatic potential, ${ }^{[12]}$ however as shown in a re- cent series whereby over one-fifth of patients had metastatic lesions, EHE is now regarded as fully malignant and not a borderline tumor. ${ }^{[13]}$

The patient presented in this report had pulmonary symptoms including worsening shortness of breath, investigations focused on ruling out respiratory causes such pneumonia and Pulmonary Tuberculosis as he also admitted to have lost significant weight. Common differential diagnosis for patients presenting with chronic respiratory symptoms include: other lung malignancies, especially non-small cell lung carcinoma and inflammatory diseases like sarcoidosis or organizing pneumonia and Tuberculosis. ${ }^{[8]}$ We made significant efforts to rule out other diagnoses. Chest x-ray suggested metastatic lesions; hence the efforts to look for other primaries elsewhere. The patient presentation with a painless scalp lesion since childhood is very unusual for EHE and was not a source of concern even to the patient despite recent increase in size; literature has documented a possibility of EHE to show malignant potential even after a long time. ${ }^{[7]}$ Due to the variability of the tumor presentation with unpredictable course, diagnosis relies on clinicopathologic characteristics and combined application of specific vascular endothelial immunohistochemical markers to differentiate it from other lesions. ${ }^{[6]}$ The use of immunohistochemistry for endothelial markers such as factor VIII-related antigen, CD34, CD31, podoplanin (D2-40 antibody) and Fli-1 is crucial to arrive at a definitive diagnosis. ${ }^{[14,15]}$

Epithelioid hemangioendothelioma being a rare diagnosis has led to the fact that no standard treatment in form of either chemotherapy or radiotherapy is available Todate other than resection. ${ }^{[16]}$ Small lesions have been resected with variable outcomes, hence surgery being recommended for small, limited lesions, ${ }^{[17]}$ We do believe that possible surgery would have made a big difference in our patient as the tumor was limited to the scalp for a long period.

In terms of different organs affected by EHE, the lungs and liver are the two organs most frequently involved, but spread through bloodstream to other sites such as bones and soft tissues is also documented. ${ }^{[8]}$ Cardiac involvement as primary EHE is very rare. ${ }^{[7,9,10]}$ We find our case to be peculiar because it involved metastasis from soft tissue to the lungs, pericardium and right atrium, we did not find other case reports in English literature of metastasis of EHE into the heart.The outcome of EHE varies depending on affected sites; solitary and skin lesions appear relatively slow-growing, whereas multicentric lesions or those with histological atypia, and lesions form sites such as bone or lungs may metastasize and carry a fatal outcome. ${ }^{[18]}$ Our patient had a very slow growing swelling over a long period of time, probably unat- 
tended the lesions transformed to a more aggressive behavior. Poor prognostic indicators include low weight, anemia, pulmonary symptoms, pleural hemorrhagic effusions and hemoptysis. ${ }^{[19,20]}$ Our patient had majority of these factors, most patients with pulmonary involvement of EHE die of respiratory failure, although death due to extrapulmonary spread may occur. ${ }^{[21]}$

In summary, we have presented a 31-year-old man who pre- sented with worsening shortness of breath of four month duration with a painless scalp lesion since his childhood, his condition worsened and he expired. Autopsy findings confirmed with immunohistochemistry assay confirmed scalp epithelioid hemangioendothelioma with metastasis to the lungs, pericardium and right atrium, the case highlights the importance of high index of suspicion and education to the community to seek medical attention for undiagnosed skin lesions.

\section{REFERENCES}

[1] Weiss SW, Enzinger FM. Epithelioid hemangioendothelioma: a vascular tumor often mistaken for a carcinoma. Cancer. 1982; 50: 970-981. http://dx.doi.org/10.1002/1097-0142(1982 0901) $50: 5<970:$ :AID-CNCR2820500527>3.0.CO;2-Z

[2] Makhlouf HR, Ishak KG, Goodman ZD. Epithelioid hemangioendothelioma of the liver: a clinicopathologic study of 137 cases. Cancer. 1999; 85(3): 562-82. http://dx.doi.org/10.1002/(SICI ) 1097-0142(19990201) 85:3<562: :AID-CNCR7>3.0.C0;2-T

[3] Mehrabi A, Kashfi AR, Fonouni H, et al. Primary malignant hepatic epithelioid hemangioendothelioma. Cancer. 2006; 107(9): 2108-21. http://dx.doi.org/10.1002/cncr. 22225

[4] Earnest F, Johnson CD. Case 96: Hepatic Epithelioid Hemangioendothelioma. Radiology. 2006; 240(1): 295-8. http://dx.doi.org /10.1148/radiol.2401032099

[5] Lau K, Masssad M, Pollak C, et al. Clinical patterns and outcome in epithelioid hemangioendothelioma with or without pulmonary involvement: insights from an internet registry in the study of a rare cancer. Chest. 2011; 140: 1312-8. PMid:21546438 [http://dx.doi.org/10.1378/chest.11-0039]

[6] Li H, Wang C, Zhu Y, et al. Epithelioid hemangioendothelioma: a clinicopathologic analysis of 13 cases. Chinese Journal of Pathology. 2015; 44(6): 386-9. PMid:26704832

[7] Agaimy A, Kaiser A, Wünsch PH. Epithelioid hemangioendothelioma of the heart in association with myelodysplastic syndrome. Z Kardiol. 2002; 91(4): 352-6. PMid:12063709 http: //dx .doi .org /10.1007/s003920200038

[8] Sugimoto T, Yamamoto K, Yoshii S. A Primary Epithelioid Hemangioendothelioma of the Right Atrium: Report of a Case and Literature Review. Open Journal of Thoracic Surgery. 2013; 3: 63-67. http://dx.doi.org/10.4236/ojts.2013.32013

[9] Marchiano D, Fisher F, Hofstetter S. Epithelioid hemangioendothelioma of the heart with distant metastasis. A case report and literature review. J Cardiovasc Surg (Torino). 1993; 34(6): 529-33.

[10] Kahlout M, Al-Mulla A, Chaikhouni A, et al. Unusual Presentation of a Rare Tumor: Cardiac epithelioid hemangioepithelioma Presenting as Cardiac Tamponade. Heart Views. 2009; 10(3): 132-135.
[11] Ramchandar N, Wojtczak HA. Pulmonary Epithelioid Hemangioendothelioma in a Patient with Crohn's Disease. Case Reports in Pediatrics. 2015; 2015: 1-3.

[12] Weiss SW, Goldblum JR. Hemangioendothelioma: vascular tumors of intermediate malignancy. In: Weiss SW, Goldblum JR, eds. Enzinger and Weiss's Soft Tissue Tumors. 5th edition. PA: Mosby. 2008: 681-702. PMid: 18843793

[13] Fletcher CDM. Diagnostic Histopathology of Tumors. Philadelphia, Pennsylvania: Elsevier Science. 2000.

[14] Fujii T, Zen Y, Sato Y, et al. Podo-planin is a useful diagnostic marker for epithelioid hemangioendo-thelioma of the liver. Mod Pathol. 2008; 21: 125-130. http://dx.doi.org/10.1038/mod pathol.3800986

[15] Gill R, O’Donnell RJ, Horvai A. Utility of immunohistochemistry for endothelial markers in distinguishing epithelioid hemangioendothelioma from carcinoma metastatic to bone. Arch Pathol Lab Med 2009; 133: 967-972.

[16] Kawachi R, Asamura H. A case of pulmonary epithelioid hemangioendothelioma. Jpn J Clin Oncol. 2010; 40: 709. http://dx.doi .org/10.1093/jjco/hyq105

[17] Schattenberg T, Kam R, Klopp M, et al. Pulmonary epithelioid hemangioendothelioma: report of three cases. Surg Today. 2008; 38: 844-9. http://dx.doi.org/10.1007/s00595-007-3712-4

[18] Shabnum A, Odell EW, Whaites E, et al. Epithelioid Hemangioendothelioma of the mandibular gingiva: case report and literature review. Int J Surg Case Rep. 2015; 14: 194-198. http: //dx.doi .o rg/10.1016/j.ijscr.2015.06.041

[19] Bagan P, Hassan M, Le Pimpec BF, et al. Prognostic factors and surgical indications for pulmonary epithelioid hemangioendothelioma: a review of the literature. Ann Thorac Surg. 2006; 82: 2010-3. PMid:17126100 http://dx.doi.org/10.1016/j .athoracsur. 2006.06.068

[20] Celikel C, Yumuk PF, Basaran G, et al. Epithelioid hemangioendothelioma with multiple organ involvement. APMIS. 2007; 115(7): 881-888. PMid:17614859 http://dx.doi.org/10.1111/j.160 0-0463.2007.apm_727.x

[21] Rosengarten D, Kramer MR, Amir G, et al. Pulmonary epithelioid hemangioendothelioma. Isr Med Assoc J. 2011; 13: 676-9. PMid:22279701 